\title{
Zwei Ansätze zur Lösung der Replikationskrise in den Wirtschaftswissenschaften
}

https://doi.org/10.1515/bfp-2019-2004

Zusammenfassung: Die Forderungen nach einem systematischen Management von Forschungsdaten steigen. Wissenschaftler haben trotzdem oftmals wenig Anreize, eine gute und auf Nachnutzbarkeit abzielende Dokumentation von Daten und ihren Forschungsprozessen zu erstellen. Wir diskutieren Data Sharing und reproduzierbare Forschung vor dem Hintergrund des Anreizschemas in der Wissenschaft und stellen zwei Ansätze der ZBW - Leibniz Informationszentrum Wirtschaft vor, die Forschende beim Data Sharing und der Publikation von Replikationen unterstützen.

Schlüsselwörter: Forschungsdaten; Replikationen; Anreize

\section{Two Approaches to Solve the Replication Crisis in Econo-} mics

\begin{abstract}
The demand for a systematic research data management is increasing. Nevertheless, incentives to document data and research in order to improve reproducibility are clearly reduced for individual researchers. In this article we discuss data sharing and reproducible research with regard to the incentive scheme in academia and present two approaches of the ZBW - Leibniz Information Centre for Economics that support researchers in data sharing and the publication of replication studies.
\end{abstract}

Keywords: Research data; replications; incentives

\section{Einleitung}

Mit der seit Jahrzehnten zunehmenden Menge und Bedeutung von Forschungsdaten, wächst auch die Notwendigkeit für deren systematisches Management. Kaum eine wissenschaftspolitische Veröffentlichung kommt derzeit ohne einen Verweis auf die Thematik Forschungsdatenmanagement aus. In ihrem Koalitionsvertrag verspricht

*Kontaktperson: Sven Vlaeminck, s.vlaeminck@zbw.eu Dr. Martina Grunow, m.grunow@zbw.eu die aktuelle Bundesregierung eine Infrastruktur zum systematischen Management wissenschaftlicher Datenbestände aufzubauen. Auch die Sicherung dieser Daten sowie der nachhaltige Zugang stehen auf der Agenda der Bundesregierung. ${ }^{1}$ Auf europäischer Ebene wird die Harmonisierung des Forschungsdatenmanagements im Rahmen der European Open Science Cloud (EOSC) vorbereitet ${ }^{2}$ und auf nationalstaatlicher Ebene die Nationale Forschungsdateninfrastruktur (NFDI) entwickelt. ${ }^{3}$ Immer mehr Universitäten verabschieden Richtlinien zum Umgang mit Forschungsdaten. ${ }^{4}$ Fachzeitschriften und Verlage beziehen Forschungsdaten zunehmend in den Begutachtungs- und Publikationsprozess von Fachartikeln ein. ${ }^{5}$

In den Wirtschaftswissenschaften sind diese Entwicklungen im Kontext der sogenannten ,Replikationskrise“ von Bedeutung. Die ZBW als weltweit größte Spezialbibliothek für die Wirtschaftswissenschaften hat in den letzten Jahren vor dem Hintergrund der Replikationskrise verstärkt Anstrengungen unternommen, um die Wirtschaftsforschung in dieser Frage zu unterstützen. Neben Informations- und Fortbildungsangeboten insbesondere für Nachwuchsforschende, ${ }^{6}$ sind zwei Informationsinfrastrukturen entstanden, die mit konkreten Lösungsansätzen einen Weg aus der Replikationskrise weisen.

\section{Hintergrund}

Replikationen dienen insbesondere der Erkenntnis, welche Ergebnisse robust und verallgemeinerbar sind und damit letztendlich der Forschung als Wegweiser und der Politik als Empfehlungen dienen können. So sagt der Präsident der Deutschen Forschungsgemeinschaft Peter Strohschneider: „Die Frage nach der Replizierbarkeit von Forschungsergeb-

$1 \mathrm{CDU} / \mathrm{CSU}$ und SPD (2018) 33.

2 European Commission (2018).

3 Rat für Informationsinfrastrukturen (2018).

4 Eine gute und aktuelle Übersicht findet sich bei forschungdaten. $\operatorname{org}(2018)$.

5 Vgl. Springer Nature (2018).

6 Vgl. http://www.zbw.eu/ueber-uns/veranstaltungen/stata/ sowie https://auffinden-zitieren-dokumentieren.de. 
nissen berührt im Kern die Frage der Qualität von Forschung und betrifft die gesamte Wissenschaft" .7

Dies zeigte sich auch in den Wirtschaftswissenschaften, wo die mangelnde Überprüfbarkeit und Replizierbarkeit von empirischen Forschungsergebnissen zu einem bedeutenden Glaubwürdigkeitsverlust der Forschung führte. ${ }^{8}$ Obwohl die Forschergemeinschaft Replikationen inzwischen weitestgehend als ein wichtiges Instrument für den wissenschaftlichen Erkenntnisprozess anerkennt, sind in der Literatur kaum Replikationsstudien zu finden. Dieser Widerspruch beruht auf einer Kombination unterschiedlicher Anreizprobleme, die verschiedene Akteure im Wissenschaftssystem betrifft. Forscher, Herausgeber von Fachzeitschriften und Drittmittelgeber bewegen sich in einem System, in welchem über Jahrzehnte weder die Überprüfbarkeit von Studien noch die Replikation von Forschungsergebnissen gefördert wurde.

Grundlage für die Replikation quantitativer Forschung ist der Zugang zu den Daten und den Analysecodes, welche in der empirischen Originalstudie verwendet wurden. Dies setzt voraus, dass die Autoren der Originalstudie dieses Material zur Verfügung und somit die Überprüfbarkeit ihrer Arbeit sicherstellen. Auf der anderen Seite müssen Wissenschaftler Zeit aufwenden, um Replikationsstudien durchzuführen und deren Ergebnisse der Forschergemeinschaft in Form von Publikationen zugänglich zu machen. In beiden Fällen spielen insbesondere Fachzeitschriften und Drittmittelgeber eine wichtige Rolle und beeinflussen die Anreize für Forscher, sowohl die Überprüfbarkeit publizierter Forschung sicherzustellen als auch Replikationen durchzuführen.

\section{Reproduzierbare Wirtschaftsforschung ist eher die Ausnahme}

Bereits 1986 hatten die Ökonomen Dewald, Thursby und Anderson versucht, die Ergebnisse von 54 im renommierten Journal of Money, Credit and Banking (JMCB) publizierten Artikeln zu replizieren. ${ }^{9}$ Nur zwei $(3,7 \%)$ der Originalstudien konnten repliziert werden. Weitere Untersuchungen ${ }^{10}$ in den nachfolgenden Jahrzehnten kamen zwar zu besseren Ergebnissen, jedoch konnten bei keiner dieser systemati-

7 DFG (2017).

8 vgl. Ioannidis und Doucouliagos (2013) sowie Bright (2013).

9 Dewald et al. (1986).

10 Vgl. z.B. McCullough (2007). schen Replikationsstudien mehr als $50 \%$ der untersuchten Studien erfolgreich repliziert werden. ${ }^{11}$

Bedeutend ist, dass ein Großteil der Originalstudien aufgrund fehlender Verfügbarkeit der zugrundeliegenden Daten und Analysecodes nicht repliziert werden konnte.

Bereits Dewald et al. diskutierten die Datenrichtlinien von Zeitschriften (Data Policies) als einen Grund für die mangelnde Überprüfbarkeit der Originalstudien. ${ }^{12}$ Data Policies bestimmen den Umgang und die Verfügbarkeit der den publizierten Artikeln zugrundeliegenden Forschungsdaten. Die Richtlinien des JMCB verpflichteten zu jener Zeit die Autoren, ihre Daten und Analysecodes auf Anfrage durch andere Forscher zur Verfügung stellen. Laut Dewald et al. reagierte ein Drittel der angeschriebenen Autoren gar nicht auf ihre wiederholten Anfragen nach diesen Daten. Ein weiteres Drittel konnte die verwendeten Programme und Daten nach eigener Angabe nicht herausgeben, hatte die Daten verloren oder verwies auf andere Quellen. Nur ein Drittel der angeschriebenen Forscher stellten Informationen zu Daten oder Analysecodes zur Verfügung. Selbst in diesen Fällen konnten viele der angegebenen Datenquellen nicht eindeutig identifiziert werden oder Dokumentationen zu den Daten fehlten und machten so eine Überprüfung unmöglich. ${ }^{13}$

\section{Data Sharing: Eine Frage der Anreize}

Das derzeitige Wissenschaftssystem setzt kaum Anreize für Forscher, Daten und Analysecodes ihrer Analysen verfügbar zu machen und somit die Überprüfbarkeit der Ergebnisse zu unterstützen. An einigen Stellen wirkt es dem sogar entgegen: Eine Forschungsdokumentation, die Dritten eine Überprüfung erlaubt, ist mit einem erhöhten Arbeitsaufwand verbunden, dem bislang keine angemessene Anerkennung gegenüber steht. Im Gegenteil, eine Veröffentlichung der verwendeten Forschungsdaten, zugehöriger Materialien und Analysecodes birgt das Risiko, dass Fehler oder Mängel in Daten oder Analyse gefunden werden, die die wissenschaftliche Reputation - ob begründet oder nicht - gefährden könnten..$^{14}$ Der hohe Publikationsdruck, welcher eine wissenschaftliche Karriere begleitet, und der Anspruch von Zeitschriften, ausschließlich innovative und neuartige Erkenntnisse zu publizieren, fördert

11 Vgl. Chang und Li (2018).

12 Dewald et al. (1986) 588.

13 Dewald et al. (1986) $589 \mathrm{f}$.

14 Duvendack et al. (2015) 171. 
zusätzlich den „Goldschatz“ der oftmals sehr zeitaufwändig erhobenen Daten zunächst selbst vollständig zu heben und den Datensatz erst sehr verzögert oder gar nicht offen zu legen.

Die drei genannten Aspekte (a) Kosten der Datenaufbereitung ohne entsprechende Honorierung, b) Sorge vor Reputationsverlusten durch mangelnde Replizierbarkeit und c) Zurückhaltungsinteresse von Daten bis zur vollständigen Verwertung offenbaren einen Zielkonflikt in der Wissenschaftspolitik. Während das „Open“-Paradigma in der Wissenschaftspolitik an Stärke gewinnt, wirken die Anreize auf Seite des individuellen Forschers der Umsetzung dieses Paradigmas entgegen. In den Wirtschaftswissenschaften ist deshalb nicht zu erwarten, dass sich das für die Überprüfbarkeit notwendige Data und Code Sharing auf freiwilliger Basis als Standard etabliert. ${ }^{15}$

Da sich trotz wissenschaftspolitischer Forderung noch kein Belohnungssystem (etwa in Form der Honorierung von Datenaufbereitung und Data Sharing etwa bei Berufungen und Evaluierungen) in der Wissenschaft durchgesetzt hat, sehen sich auch Forschungsförderer und Fachzeitschriften zunehmend in der Position, ihre Anforderungen in Bezug auf Datenzugang und Datendokumentation bei Projekten einzufordern, die mit öffentlichen Mittel gefördert werden.

Während die Deutsche Forschungsgemeinschaft (DFG) noch verhalten mit der Verpflichtung zum Data Sharing bei der Beantragung von Drittmittelprojekten verfährt, ${ }^{16}$ werden Drittmittelnehmer etwa beim 8. Forschungsrahmenprogramm der EU, besser bekannt als Horizon 2020, deutlich stärker in die Pflicht genommen. ${ }^{17}$

Auch bei den Fachzeitschriften in der Wirtschaftsforschung scheinen sich Vorgaben in Bezug auf die Datenverfügbarkeit langsam durchzusetzen. Inzwischen besitzen laut einer Untersuchung aus dem Jahr 2015 gut 14 \% von 350 untersuchten Journals in den Wirtschaftswissenschaften entsprechende Richtlinien zur Verfügbarkeit von Daten und Analysecodes. ${ }^{18}$ Allerdings verfügen nur rund $8 \%$ der im Social Science Citation Index $(\mathrm{SSCI})^{19}$ gelisteten

15 Vgl. Andreoli-Versbach und Mueller-Langer (2013) sowie MuellerLanger und Andreoli-Versbach (2018).

16 Die DFG verlangt bis heute keine verpflichten Datenmanagementpläne, sondern führt aus, dass „die Antragstellenden daher ausführen [sollten], welche Forschungsdaten im Verlauf eines wissenschaftlichen Forschungsvorhabens entstehen."Vgl. DFG (2015) 1.

17 Vgl. https://ec.europa.eu/research/participants/docs/h2020-funding-guide/cross-cutting-issues/open-access-data-management/data-management_en.htm.

18 Vgl. Vlaeminck und Herrmann (2015).

19 Vgl. http://mjl.clarivate.com/cgi-bin/jrnlst/jlresults.cgi?PC=SS\& Alpha $=$ A.
Zeitschriften über eine verpflichtende Data Policy, was zeigt, dass auch auf Seiten der Fachzeitschriften noch Hürden bestehen..$^{20}$

Gespräche mit verschiedenen Herausgebern legen nahe, dass viele Fachzeitschriften die Kosten der Einführung entsprechender Richtlinien scheuen. Befürchtungen bestehen insbesondere in Bezug auf den erwarteten Aufwand bei der Implementierung einer solchen Policy und der Überprüfung, ob die Vorgaben auch eingehalten werden. Auch bestehen Sorgen vor einem hohen Aufwand hinsichtlich der Kommunikation mit den Autoren, sowie der Etablierung und Pflege eines zugehörigen Datenarchivs. Darüber hinaus existieren Befürchtungen vor einem Rückgang an Einreichungen, sollten die Anforderungen an das $\mathrm{Pu}$ blizieren im Journal durch die Einführung einer entsprechenden Data Policy steigen.

Durch die veränderten Voraussetzungen zur Publikation von Artikeln und den Bezug von Drittmitteln, steigen die Anreize für Forscher Datenmanagement, Datendokumentation und Datenzugang entsprechend diesen Richtlinien zu erarbeiten.

\section{Das ZBW Journal Data Archive}

Um Zeitschriften bei der Umsetzung ihrer Datenrichtlinien zu unterstützen, wurde Ende 2016 mit dem Datenrepositorium ZBW Journal Data Archive (JDA) ein kostenfreier Service für wirtschaftswissenschaftliche Fachzeitschriften gestartet. Forschern wird durch das JDA zudem eine niedrigschwellige und einfache Infrastruktur an die Hand zur Verfügung gestellt, mit welcher sie die Anforderungen entsprechender Data Policies von Drittmittelgebern erfüllen können.

Das JDA wurde in der Zeit zwischen 2011 und 2016 im DFG-geförderten Projekt EDaWaX gemeinsam mit dem Herausgeberkreis einer Fachzeitschrift aus den Wirtschaftswissenschaften auf Basis der Open Source Software CKAN entwickelt.

Teilnehmende Fachzeitschriften können diese Infrastruktur als Datenarchiv nutzen. Dazu registrieren die Redaktionen zunächst den corresponding author von empirischen Artikeln im System. Das JDA informiert dann den Autoren über die erfolgte Registrierung und fordert zum Setzen eines Passworts für den eigenen Account auf. Ist dies geschehen, kann mit dem Upload der Replikationsdateien begonnen werden. Zunächst werden allgemeine Metadaten vom System abgefragt (wie etwa die Namen der

20 Vgl. Hoeffler (2017). 
Autoren, der Titel der Dateneinreichung, Klassifikationen usw.) darunter alle Pflichtfelder des DataCite-Metadatenschemas. $^{21}$

In den nächsten Schritten können verschiedene Materialien ins Datenarchiv überführt werden, wobei der Autor stets eine Auswahl trifft, welche Art von Material hochgeladen wird. Dies können Datensätze, Analysecodes, Dokumentation oder weitere Daten sein. Abhängig von der gewählten Art der zu übermittelnden Daten verändert sich auch das Metadatenformular, was zu dieser Art von Materialien ausgefüllt werden soll. Für Datensätze werden mehr Metadaten erhoben als für andere Materialarten.

Nach Abschluss des Uploadprozesses werden die Daten im System gespeichert und stehen den Redaktionen für Reviewprozesse zur Verfügung. Nach erfolgreicher Prüfung der Daten und der Veröffentlichung des zugehörigen Artikels, ergänzen die Redaktionen einige bibliografischen Angaben (insbesondere den DOI zum Artikel) im System und schalten die Dateneinreichung frei. Im Falle noch fehlender Daten oder zu verbessernder Dokumentation können die Redaktionen direkt aus der Applikation heraus mit den Autoren in Kontakt treten und entsprechende Nachbesserungen vor einer Veröffentlichung der Daten anmahnen.

Ist die Einreichung komplett, erhalten die Daten einen DOI und sind so eindeutig referenzier- und zitierbar. Gerade durch die Zitierbarkeit der Daten wird ein Beitrag zur Anerkennung der geleisteten Arbeit der Autoren in Bezug auf die Datenbereitstellung und deren Dokumentation geleistet.

Für Fachzeitschriften liegt der zentrale Nutzen des JDA darin, dass diese vom Aufbau und der Pflege eines Datenarchivs befreit sind, die Kommunikation mit Autoren vereinfacht und Support durch das JDA angeboten wird. Dadurch, dass die Autoren die Dateneingabe und Übermittlung im Regelfall selbst vollziehen, können die Ressourcen der Redaktionen für andere Aufgaben eingesetzt werden.

Bis Mitte 2018 nutzen bereits vier Fachzeitschriften aus der Wirtschaftsforschung das JDA als Datenarchiv. Drei dieser Zeitschriften verfügen über einen ,Impact Factor und zählen zu den wichtigsten wirtschaftswissenschaftlichen Journalen, die in Deutschland publiziert werden.

21 DataCite Metadata Working Group (2017).

\section{Von der Überprüfbarkeit zur Replikation}

Stehen alle für eine Replikation notwendigen Informationen zur Verfügung, bedarf es als nächstes des Versuches, die Ergebnisse der entsprechenden Originalstudie zu replizieren und im weiteren Schritt diese Replikationsstudie zu publizieren.

Mueller-Langer et al. (2018) zeigen, dass nur 0,1\% aller zwischen 1974 und 2014 in den Top-50-Zeitschriften publizierten Artikel Replikationen waren. Duvendack et al. (2015) fanden, dass im Jahr 2013 nur rund $2 \%$ von allen im SSCI gelisteten wirtschaftswissenschaftlichen Fachzeitschriften überhaupt Replikationsstudien publizieren.

Die veröffentlichten Replikationsstudien weisen zudem eine klare Tendenz in ihren Ergebnissen auf: Sie können die Ergebnisse der Originalstudie nicht bestätigen. Die selektive Publikation bestimmter Ergebnisse wird als ,publication bias' bezeichnet.

Die daraus abzuleitende verschwindend geringe $\mathrm{Pu}$ blikationschance für Replikationsstudien ist Kern einer weiteren Anreizproblematik. Eine Replikationsstudie birgt für den individuellen Wissenschaftler schon vor deren Durchführung das Risiko, dass diese gar nicht oder aufgrund des publication bias nur bei negativem Ausgang des Replikationsversuchs veröffentlicht wird.

Auf Seiten der Fachzeitschriften, bei welchen der ,Innovations- und Originalitätsanspruch' an ihre Publikationen vermeintlich gegen eine Veröffentlichung von erfolgreichen Replikationen spricht, mag ein weiterer Aspekt negative Anreize für die Publikation von Replikationsstudien setzen: Kann ein Replikationsversuch die veröffentlichten Ergebnisse einer Originalstudie nicht bestätigen, dann kann das auch Fragen an die Qualität des Begutachtungsprozesses und der Publikationen in der betroffenen Zeitschrift aufwerfen. Hinzu kommt, dass insbesondere Replikationsstudien, welche die Originalstudie bestätigen, weniger häufig als Originalstudien zitiert werden. Dies könnte ggf. zu einem verringerten Impact Factor führen.

In der vorherrschenden ,publish or perish'-Kultur, in welcher die Anzahl der Publikationen ein entscheidendes Kriterium für den Karriereweg in der Wissenschaft ist, sind Aufwand und Risiko einer Replikationsstudie im Verhältnis zum erwarteten Nutzen einer Publikation somit zu groß und Anreize, Replikationen durchzuführen, sind drastisch reduziert.

Hinzu kommt der paradoxe Ruf von Replikationsstudien. Während einerseits ein überwiegender Teil der Forschenden zustimmt, dass Replikationen ein notwendiges Instrument zur Prüfung der Belastbarkeit und Verallgemei- 
nerbarkeit empirischer Ergebnisse sind, ${ }^{22}$ wird Replikationsstudien andererseits ein wissenschaftlicher Zusatznutzen bzw. Erkenntnisgewinn abgesprochen, da „nur“ ein bereits publizierter Forschungsprozess erneut durchlaufen würde. ${ }^{23}$ Auch deshalb wird Forschern, die Replikationen durchführen, oft eine eigenständige wissenschaftliche Leistung abgesprochen. Im schlimmeren Fall werden sie als Querulanten angesehen, die ihre eigene wissenschaftliche Karriere durch das Aufdecken von Fehlern anderer Forschern voranbringen würden. ${ }^{24}$

Mit der internationalen Fachzeitschrift „International Journal for Re-Views in Empirical Economics“ (IREE) haben die ZBW und deren Projektpartner (die Leuphana Universität Lüneburg, das DIW Berlin und das IZA Bonn) ein Konzept entwickelt, welches in dieser komplexen Struktur die Anreize derart verschiebt, dass die Durchführung von Replikationsstudien gefördert wird. Erklärtes Ziel von IREE ist es, Replikationen auch in der wissenschaftlichen Praxis ihren Stellenwert als Instrument im wissenschaftlichen Erkenntnisprozess zu verschaffen.

IREE publiziert Replikationsstudien unabhängig von ihrem Ergebnis, d.h. sowohl erfolgreiche als auch gescheiterte Replikationsversuche werden veröffentlicht. Dies minimiert das ex-ante Risiko bezüglich des Ausgangs der Studie für replizierende Forscher mit Hinblick auf den publication bias. Das peer-review-Begutachtungsverfahren für jede Veröffentlichung in IREE schließt auch immer die zugrundeliegenden Daten, Datenzugangsbeschreibung und den Analysecode ein.

Alle Publikationen in IREE sind zitierfähig. Jeder Teil einer Publikation - Manuskript, Programmierung und Datensatz - erhält eine jeweils eigene DOI. Dies ermöglicht eine veränderte Zitierweise, in welcher eine Originalstudie immer gemeinsam mit ihrer Replikation zitiert wird. Auf diese Weise, steigt für den replizierenden Forscher der erwartete Nutzen einer Replikationsstudie mit Hinblick auf die Anerkennung als (zitierte) Publikation. Durch die gleichwertige Veröffentlichung von erfolgreichen und gescheiterten Replikationen zeigt IREE, dass Replikationen nicht der Aufdeckung von Fehlern konkurrierender Forscher, sondern vielmehr als Wegweiser für den wissenschaftlichen Erkenntnisprozess dienen.

Neben Replikationsstudien veröffentlicht IREE Arbeiten, die sich mit der Entwicklung von Methoden und Standards von Replikationen in den Wirtschaftswissenschaften beschäftigen. Zudem werden Datensätze und Beschrei-

22 Fecher et al. (2016) 9.

23 Vgl. Park (2004) 194.

24 Vgl. Longo und Drazen (2016) 276. bungen von Datensätzen veröffentlicht. So fördert IREE die bisher mangelnde Anerkennung für den Aufwand der Beschreibung und der Veröffentlichung von Datensätzen. Sind diese als zitierfähige Publikation verfügbar, steigen auch hier die Anreize für den individuellen Forscher, seine Daten bereitzustellen.

Sämtliche Publikationen in IREE sind im Open Access verfügbar und werden durch EconStor ${ }^{25}$ und $\mathrm{RePEc}^{26}$ international verbreitet und die Datensätze und Programmierungen werden im ZBW Journal Data Archive dauerhaft gespeichert und zugänglich gemacht. Zudem verzichtet IREE vollständig auf Publikationsgebühren für Autoren.

Mit dem inhaltlichen Schwerpunkt auf Replikationen und der konsequenten Umsetzung von Open-Access-Standards ist IREE derzeit einzigartig im Markt wirtschaftswissenschaftlicher Fachzeitschriften positioniert und fördert den Kulturwandel hin zu einer transparenten und offenen Wissenschaft, wie sie von Wissenschaft und Politik gefordert wird.

\section{Fazit}

Die Anforderungen und die Notwendigkeit für ein systematisches Management von Forschungsdaten steigen. Insbesondere Forschungsförderer basieren ihre Entscheidungen in der Drittmittelvergabe zunehmend auf Datenmanagementplänen und der Sicherstellung des Zugangs zu den Forschungsdaten. Diese Anforderungen gehen aber nicht mit einer entsprechenden Honorierung der geleistesten Arbeit der Forschenden einher. Somit haben Wissenschaftler oftmals wenig Anreize, eine gute und auf Nachnutzbarkeit abzielende Dokumentation von Daten und ihren Forschungsprozessen mit den Daten zu erstellen. Dies führt zu einer fehlenden Überprüfbarkeit von empirischen Forschungsergebnissen. Obwohl Replikationen als wichtiges Instrument im wissenschaftlichen Erkenntnisprozess anerkannt sind, werden im Kontext der Replikationskrise in den Wirtschaftswissenschaften die mangelnde Anzahl an Replikationsstudien und die geringe Replizierbarkeit von publizierten Ergebnissen diskutiert. Ebenso wie in der Dokumentation von Forschungsdaten

25 EconStor ist eines der größten wirtschaftswissenschaftlichen Open-Access-Volltextrepositories. Siehe auch https://www.econstor. eu.

26 Research Papers in Economics (RePEc) ist eine bottom-up-Initiative aus der Wissenschaft, deren Zweck ein zentrales Nachweisportal aller Arten von ökonomischen Publikationen (Working Paper, Preprints, Zeitschriftenartikel aber auch Softwarekomponenten) ist. Siehe auch http://repec.org. 
liegt hier ein Anreizproblem vor, welches auf mangelnder Anerkennung von Replikationsstudien beruht.

Mit zwei unterschiedlichen und disziplinären Ansätzen greift die ZBW diese Problematik auf und stellt unterstützende Infrastrukturen zur Verfügung, die die unterschiedlichen Anforderungen von Drittmittelgebern, Fachzeitschriften und Forschern hinsichtlich transparenter und nachvollziehbarer Forschung harmonisieren. Das Journal Data Archive bietet Fachzeitschriften eine Infrastruktur für ihre Data Policies und leistet durch die Vergabe von zitierfähigen DOIs für Forschungsdaten und weiteren Materialien Anreize für Data Sharing und Open Science. Das International Journal for Re-Views in Empirical Economics (IREE) bietet Forschenden die Möglichkeit, Replikationsstudien zu publizieren. IREE fördert somit als Fachzeitschrift für Replikationen in den Wirtschaftswissenschaften den kulturellen Wandel hinsichtlich transparenter, nachvollziehbarer und qualitativ hochwertiger Forschungsergebnisse.

\section{Literaturverzeichnis}

Andreoli Versbach, Patrick; Mueller-Langer, Frank (2013): Open Access to Data: An Ideal Professed but Not Practised. In: RatSWD Working Paper Series Nr. 215. Max Planck Institute for Intellectual Property \& Competition Law Research Paper Nr. 13-07. doi:10.2139/ssrn.2224146.

Bright, Peter (2013): Microsoft Excel: The ruiner of global economies? A paper used to justify austerity economics appears to contain an Excel error. Blogbeitrag. Arctechnica. Verfügbar unter https://arstechnica.com/tech-policy/2013/04/microsoft-excelthe-ruiner-of-global-economies.

CDU/CSU und SPD (2018): Ein neuer Aufbruch für Europa. Eine neue Dynamik für Deutschland. Ein neuer Zusammenhalt für unser Land. Koalitionsvertrag zwischen CDU, CSU und SPD. 19. Legislaturperiode, Berlin. Verfügbar unter https://www.bundesregierung.de/Content/DE/_Anlagen/2018/03/2018-03-14-koalitionsvertrag.pdf.

Chang, Andrew C.; Li, Philipp (2018): Is Economics Research Replicable? Sixty Published Papers from Thirteen Journals Say, Usually Not'. In: Critical Finance Review, 7(2), doi: 10.1561/ 104.00000053.

DataCite Metadata Working Group (2017): DataCite Metadata Schema for the Publication and Citation of Research Data. Version 4.1. DataCite e.V. doi:10.5438/0015.

Dewald, William G.; Thursby, Jerry G.; Anderson, Richard G. (1986): Replication in empirical economics: The journal of money, credit and banking project. In: American Economic Review, 76 (4), 587-603.

DFG (2015): Leitlinien zum Umgang mit Forschungsdaten. Verfügbar unter http://www.dfg.de/download/pdf/foerderung/antragstellung/forschungsdaten/richtlinien_forschungsdaten.pdf.

DFG (2017): DFG-Stellungnahme zur Replizierbarkeit von Forschungsergebnissen. Information für die Wissenschaft Nr. 18 vom
25. April 2017. Verfügbar unter http://www.dfg.de/foerderung/ info_wissenschaft/2017/info_wissenschaft_17_18/.

Duvendack, Maren; Palmer-Jones, Richard; Reed, W. Robert (2015):

Replications in Economics: A Progress Report. Econ Journal

Watch. Scholarly Comments on Academic Economics, 12 (2), 164-91.

European Commission (2018): EOSC Strategic Implementation. Roadmap 2018-2020. Verfügbar unter https://ec.europa.eu/ research/openscience/pdf/eosc_strategic_implementation_roadmap_large.pdf.

Fecher, Benedikt; Fräßdorf, Mathis; Wagner, Gert G. (2016): Perceptions and Practices of Replication by Social and Behavioral Scientists: Making Replications a Mandatory Element of Curricula Would Be Useful, IZA Discussion Papers, No. 9896, Institute for the Study of Labor (IZA), Bonn. Verfügbar unter http://hdl. handle.net/10419/141655.

Forschungsdaten.org (2018): Institutionelle Policies. Verfügbar unter http://www.forschungsdaten.org/index.php/Data_Policies\#Institutionelle_Policies.

Hoeffler, Jan H. (2017): Replication and Economics Journal Policy. In: American Economic Review, 107 (5), 52-55. doi:10.1257/aer. p20171032.

Ioannidis, John; Doucouliagos, Chris (2013): What's to know about the credibility of empirical economics? In: Scientific credibility of economics. Journal of Economic Surveys, 27 (5), 997-1004, doi:10.1111/joes.12032.

Longo, Dan; Drazen, Jeffrey M. (2016): Data Sharing. In: New England Journal of Medicine, 374 (3), 276-77, doi:10.1056/ NEJMe1516564.

McCullough, Bruce D. (2007): Got Replicability? In: The Journal of Money, Credit and Banking Archive. Econ Journal Watch: Scholarly Comments on Academic Economics, 4 (3), 326-37.

Mueller-Langer, Frank; Andreoli-Versbach, Patrick (2018): Open access to research data: Strategic delay and the ambiguous welfare effects of mandatory data disclosure. In: Information Economics and Policy, (42), 20-34, doi: 10.1016/j.infoecopol.2017.05.004.

Park, Caroline L. (2004): What is the value of replicating other studies? In: Research Evaluation, 13 (3), 189-95, doi:10.3152/ 147154404781776400.

Rat für Informationsinfrastrukturen (2018): Zusammenarbeit als Chance. Zweiter Diskussionsimpuls zur Ausgestaltung einer Nationalen Forschungsdateninfrastruktur (NFDI) für die Wissenschaft in Deutschland. Verfügbar unter http://www.rfii.de/? wpdmdl $=2529$.

Springer Nature (2018): Research Data Support. Verfügbar unter https://www.springernature.com/gp/authors/research-datapolicy.

Vlaeminck, Sven; Herrmann, Lisa-Kristin (2015): Data Policies and Data Archives: A New Paradigm for Academic Publishing in Economic Sciences? In: New Avenues for Electronic Publishing in the Age of Infinite Collections and Citizen Science: Scale, Openness and Trust. Proceedings of the 19th International Conference on Electronic Publishing, hg. v. Birgit Schmidt und Milena Dobreva. IOS Press, Amsterdam, 145-55, doi:10.3233/978-161499-562-3-145. 


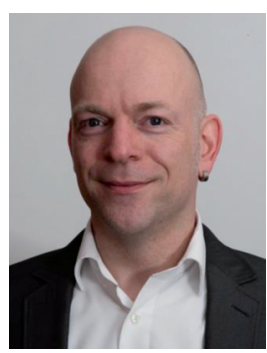

Sven Vlaeminck

Leibniz-Informationszentrum Wirtschaft

(ZBW)

Neuer Jungfernstieg 21

D-20354 Hamburg

s.vlaeminck@zbw.eu

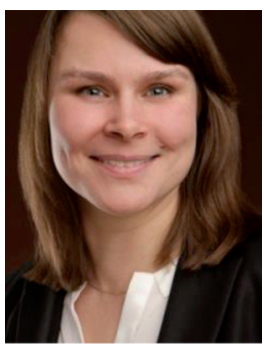

\section{Dr. Martina Grunow}

Leibniz-Informationszentrum Wirtschaft (ZBW)

Neuer Jungfernstieg 21

D-20354 Hamburg

m.grunow@zbw.eu 